\title{
On the Regularized Determinant for Non-Invertible Elliptic Operators
}

\author{
R. E. Gamboa-Saraví, M. A. Muschietti, and J. E. Solomin
}

Facultad de Ciencias Exactas, Universidad Nacional de La Plata, Comisión de Investigaciones Científicas de la Provincia de Buenos Aires, 1900 La Plata, Argentina

\begin{abstract}
We propose a technique for regularizing the determinant of a noninvertible elliptic operator restricted to the complement of its nilpotent elements. We apply this approach to the study of chiral changes in the fermionic path-integral variables.
\end{abstract}

\section{Introduction}

In the computation of quadratic path-integrals one is naturally led to the evaluation of determinants of differential operators. These determinants clearly diverge because the eigenvalues $\lambda_{j}$ increase without bound. Therefore, it is necessary to adopt some regularization procedure. One technique which has proved to be very useful is the $\zeta$-function regularization [1]. Given an elliptic invertible operator $D$ of order $m>0$, defined on a compact manifold $M$ without boundary, of dimension $n$, one forms a generalized $\zeta$-function from $D$ by defining

$$
\zeta(s, D)=\sum_{j}\left\langle D^{-s} \phi_{j}, \phi_{j}\right\rangle,
$$

where $\left\{\phi_{j}\right\}$ is any orthonormal basis and $D^{-s}$ is defined following Seeley [2]. For a normal $D$, we can take its eigenfunctions as $\phi_{j}$ 's, and then (1.1) becomes

$$
\zeta(s, D)=\sum_{j} \lambda_{j}^{-s} \text {. }
$$

These series converge only for $\operatorname{Re} s>n / m$, but $\zeta(s, D)$ can be analytically extended to a meromorphic function of $s$ in the whole complex plane [2]. In particular, it is regular at $s=0$.

We can define the regularized determinant of $D$, $\operatorname{Det}(D)$, as

$$
\operatorname{Det}(D)=\left.\exp \left(-\frac{d \zeta}{d s}(s, D)\right)\right|_{s=0} .
$$

Note that, for a normal $D$, since the $\zeta$-function is given by Eq. (1.2), its derivative at $s=0$ is formally equal to $-\sum_{j} \ln \lambda_{j}$, and then Eq. (1.3) turns to be the regularization of the product of the eigenvalues of $D$. 
The previous definition, (1.3), holds only for invertible operators. Otherwise, i.e. when $D$ admits $\lambda=0$ as eigenvalue, any natural definition for its determinant should vanish. However, in many interesting applications, the evaluation of the determinant $\operatorname{Det}^{\prime}(D)$ of the restriction of $D$ to a suitable subspace where it is invertible, turns out to be necessary. This occurs, for instance, when studying fermion fields coupled to a gauge field in a non-trivial topological sector.

In a previous work [3], we studied this problem for normal operators, by defining

$$
\operatorname{Det}^{\prime}(D)=\lim _{\alpha \rightarrow 0^{+}} \frac{\operatorname{Det}(D+\alpha I)}{\alpha^{N}}
$$

where $\alpha$ is a real parameter, and $N=\operatorname{dim} \operatorname{Ker} D$.

The aim of this paper is the generalization of this definition to the general case. As it is discussed in Sect. 2, this generalization is not straightforward owing to the fact that the orthogonal complement of the null space of $D$ is not, in general, a stable subspace for $D$.

In Sect. 3, we apply this approach to the study of the Jacobian arising from a chiral change in the fermionic variables in the path-integral defining the generating functional for a fermionic field coupled to arbitrary external fields.

\section{A Regularized Determinant of a Non-Invertible Elliptic Operator}

Let $D$ be an elliptic ${ }^{1}$ operator of order $m>0$ acting on the sections of a vector bundle $\mathscr{F}$ over a $n$-dimensional compact manifold $M$ without boundary.

We shall denote by $\mathscr{N}$ the set $\bigcup_{k \in \mathbb{N}} \operatorname{Ker}\left(D^{k}\right)$.

Lemma 1. $\mathcal{N}$ is a finite dimensional space.

Proof. For an eigenvalue $\lambda$ of $D$ we shall write $j_{D}(\lambda)=\operatorname{dim} \bigcup_{k \in \mathbb{N}} \operatorname{Ker}(D-\lambda)^{k}$.

Let $\alpha$ be a positive real number such that $L=D+\alpha I$ is invertible. For $l \in \mathbb{N}$ large enough, $L^{-l}$ is a Hilbert-Schmidt operator. So $\sum_{|\lambda|=\alpha^{-l}} j_{L^{-l}}(\lambda)$ is finite, see for
instance [4]. Then

$$
\operatorname{dim} \mathscr{N}=\sum_{\lambda=0} j_{D}(\lambda) \leqq \sum_{|\lambda|=\alpha} j_{L}(\lambda)<\infty . \quad \text { Q.E.D. }
$$

We shall denote:

$$
\begin{aligned}
N & =\operatorname{dim} \mathscr{N}, \\
P & =\text { orthogonal projection onto } \mathscr{N}, \\
{ }^{\perp} D & =(I-P) D .
\end{aligned}
$$

Note that, since $\mathscr{N}$ is a finite dimensional subspace of smooth sections of $\mathscr{F}, P$ is an infinitely smoothing pseudo-differential operator (see for instance [5]); thus the principal symbols of $D, D+P,{ }^{\perp} D+P$, and ${ }^{\perp} D$ coincide, and then all of them are elliptic operators.

1 Throughout this paper we assume that the elliptic operator $D$ has a ray of minimal growth (see [2]) 
Lemma 2. a) The operators $D+P$ and ${ }^{\perp} D+P$ are invertible and their eigenvalues coincide.

b) For $\alpha>0$ small enough, the operators $D+\alpha I$ and ${ }^{\perp} D+\alpha I$ are invertible and their eigenvalues coincide.

Proof. For a $\phi$ such that $(D+P) \phi=0$, let us take $\phi=\phi_{1}+\phi_{2}$, with $\phi_{1} \in \mathscr{N}^{\perp}$ and $\phi_{2} \in \mathcal{N}$. Then, we have $D \phi=-\phi_{2}$, so $\phi=\phi_{2} \in \mathscr{N}$, since $D \phi \in \mathscr{N}$. This implies $D^{k} \phi_{2}=(-1)^{k} \phi_{2}=0$ for some $k$.

The invertibility of ${ }^{\perp} D+P$ is evident.

It is easy to see that if $\lambda$ is an eigenvalue of $D+P$, it is also an eigenvalue of ${ }^{\perp} D+P$.

Now, if $\lambda$ is such that there exists $\phi \neq 0$ satisfying $\left({ }^{\perp} D+P\right) \phi=\lambda \phi$, we can again write $\phi=\phi_{1}+\phi_{2}$, with $\phi_{1} \in \mathscr{N}^{\perp}$ and $\phi_{2} \in \mathscr{N}$.

If $\lambda \neq 1,{ }^{\perp} D\left(\phi_{1}+\phi_{2}\right)=\lambda \phi_{1}+(\lambda-1) \phi_{2}$, then $\phi_{2}=0$, and ${ }^{\perp} D \phi_{1}=\lambda \phi_{1}$, so $D \phi_{1}=\lambda \phi_{1}+\psi$. For $k$ such that $D^{k} \psi=0$, the function

$$
\phi_{1}+\sum_{j=1}^{k} \frac{D^{j-1}}{(\lambda-1)^{j}} \psi
$$

is an eigenfunction of $D+P$ with eigenvalue $\lambda$.

It is clear that if $\lambda=1$ is an eigenvalue of ${ }^{\perp} D+P$ then it is also an eigenvalue for $D+P$.

We can prove analogously b). Q.E.D.

We shall choose an orthonormal basis $\left\{\phi_{j}\right\}_{j=1, \ldots, N}$ of $\mathscr{N}$ such that if $\phi_{j} \notin \operatorname{Ker}\left(D^{k}\right)$, then $\phi_{j} \perp \operatorname{Ker}\left(D^{k}\right)$. And any orthonormal basis $\left\{\phi_{j}\right\}_{j>N}$ of $\mathscr{N}^{\perp}$.

Lemma 3. For $\alpha>0$ small enough and $\operatorname{Re}(-s)$ sufficiently large, we have for $n \in \mathbb{N}$

a) $\left\langle(D+\alpha I)^{s} \phi_{n}, \phi_{n}\right\rangle=\left\langle\left({ }^{\perp} D+\alpha I\right)^{s} \phi_{n}, \phi_{n}\right\rangle$,

b) $\left\langle(D+P) \phi_{n}, \phi_{n}\right\rangle=\left\langle\left({ }^{\perp} D+P\right) \phi_{n}, \phi_{n}\right\rangle$,

c) $\left\langle(D+P+\alpha I)^{s} \phi_{n}, \phi_{n}\right\rangle=\left\langle\left({ }^{\perp} D+P+\alpha I\right)^{s} \phi_{n}, \phi_{n}\right\rangle$,

and, if $n>N,\left\langle(D+P+\alpha I)^{s} \phi_{n}, \phi_{n}\right\rangle=\left\langle(D+\alpha I)^{s} \phi_{n}, \phi_{n}\right\rangle$.

Proof. a) Since $D+\alpha I$ and ${ }^{\perp} D+\alpha I$ have the same eigenvalues (Lemma 2) we can choose a curve $\Gamma$ in the $s$-complex plane such that for $\operatorname{Re} s<0$ [2]

$$
(D+\alpha I)^{s} \phi=\frac{i}{2 \pi} \int_{\Gamma} \lambda^{s}(D+(\alpha-\lambda) I)^{-1} \phi d \lambda,
$$

and

$$
\left({ }^{\perp} D+\alpha I\right)^{s} \phi=\frac{i}{2 \pi} \int_{\Gamma} \lambda^{s}\left({ }^{\perp} D+(\alpha-\lambda) I\right)^{-1} \phi d \lambda .
$$

Then, it is enough to prove that

$$
\left\langle(D+(\alpha-\lambda) I)^{-1} \phi_{n}, \phi_{n}\right\rangle=\left\langle\left({ }^{\perp} D+(\alpha-\lambda) I\right)^{-1} \phi_{n}, \phi_{n}\right\rangle .
$$

If $n \leqq N$,

$$
(D+(\alpha-\lambda) I)^{-1} \phi_{n}=\sum_{j=0}^{N} \frac{(-1)^{j} D^{j}}{(\alpha-\lambda)^{j+1}} \phi_{n},
$$

so

$$
\left\langle(D+(\alpha-\lambda) I)^{-1} \phi_{n}, \phi_{n}\right\rangle=\frac{1}{\alpha-\lambda}=\left\langle\left({ }^{\perp} D+(\alpha-\lambda) I\right)^{-1} \phi_{n}, \phi_{n}\right\rangle .
$$


If $n>N$ and $\psi_{n}=(D+(\alpha-\lambda) I)^{-1} \phi_{n}$, we can write $\psi_{n}=\psi_{n}^{\prime}+\psi_{n}^{\prime \prime}$, with $\psi_{n}^{\prime} \in \mathcal{N}^{\perp}$ and $\psi_{n}^{\prime \prime} \in \mathscr{N}$, then

$$
D \psi_{n}^{\prime}=\phi_{n}-(\alpha-\lambda) \psi_{n}^{\prime}-(D+(\alpha-\lambda) I) \psi_{n}^{\prime \prime},
$$

so ${ }^{\perp} D \psi_{n}^{\prime}=\phi_{n}-(\alpha-\lambda) \psi_{n}^{\prime}$. Therefore

$$
\left\langle(D+(\alpha-\lambda) I)^{-1} \phi_{n}, \phi_{n}\right\rangle=\left\langle\psi_{n}^{\prime}, \phi_{n}\right\rangle=\left\langle\left({ }^{\perp} D+(\alpha-\lambda) I\right)^{-1} \phi_{n}, \phi_{n}\right\rangle .
$$

Assertions b) and c) can be proved analogously. Q.E.D.

Given an invertible elliptic operator $L$, and $s$ such that $\operatorname{Re} s$ is large enough, the complex power $L^{-s}$ [2] is a trace-class operator. We can define the $\zeta$-function as

$$
\zeta(s, L)=\operatorname{Tr}\left[L^{-s}\right]=\sum_{n}\left\langle L^{-s} \phi_{n}, \phi_{n}\right\rangle=\int \operatorname{tr}\left[K_{-s}(L ; x, x)\right] d x,
$$

where $K_{-s}(L ; x, x)$ is the kernel of the operator $L^{-s}$ evaluated at $x=y$. The $\zeta$-function can be meromorphically extended to the whole $s$-plane, and it is regular at $s=0$. The regularized determinant of the operator $L$ is defined as

$$
\operatorname{Det}(L)=\left.\exp \left\{-\frac{d \zeta}{d s}(s, L)\right\}\right|_{s=0} .
$$

For $N, P$, and ${ }^{\perp} D$ as in (2.1), we have the following theorem:

\section{Theorem.}

$$
\lim _{\alpha \rightarrow 0^{+}} \frac{\operatorname{Det}(D+\alpha I)}{\alpha^{N}}=\operatorname{Det}(D+P)=\operatorname{Det}\left({ }^{\perp} D+P\right) .
$$

Proof. For Res large enough, from Lemma 3, we can write

$$
\begin{aligned}
\zeta(s, D+\alpha I) & =\sum_{n=1}^{N}\left\langle(D+\alpha I)^{-s} \phi_{n}, \phi_{n}\right\rangle+\sum_{n=N+1}^{\infty}\left\langle(D+\alpha I)^{-s} \phi_{n}, \phi_{n}\right\rangle \\
& =N \alpha^{-s}+\sum_{n=N+1}^{\infty}\left\langle(D+\alpha I)^{-s} \phi_{n}, \phi_{n}\right\rangle,
\end{aligned}
$$

and analogously

$$
\zeta(s, D+P+\alpha I)=N(1+\alpha)^{-s}+\sum_{n=N+1}^{\infty}\left\langle(D+\alpha I)^{-s} \phi_{n}, \phi_{n}\right\rangle .
$$

Therefore

$$
\zeta(s, D+\alpha I)-N \alpha^{-s}=\zeta(s, D+P+\alpha I)-N(1+\alpha)^{-s} .
$$

Since both terms can be analytically extended to a neighbourhood of $s=0$, and from the continuity established in ref. [3], we have

$$
\lim _{\alpha \rightarrow 0^{+}} \frac{\operatorname{Det}(D+\alpha I)}{\alpha^{N}}=\operatorname{Det}(D+P) .
$$

Moreover, also from Lemma 3, we get $\zeta(s, D+P)=\zeta\left(s,{ }^{\perp} D+P\right)$, and then

$$
\operatorname{Det}(D+P)=\operatorname{Det}\left({ }^{\perp} D+P\right) \text {. Q.E.D. }
$$

This theorem allows us to give the following definition of the determinant of the restriction of $D$ to $\mathscr{N}^{\perp}$. 
Definition.

$$
\operatorname{Det}^{\prime}(D) \equiv \lim _{\alpha \rightarrow 0^{+}} \frac{\operatorname{Det}(D+\alpha I)}{\alpha^{N}}
$$

Note that for an invertible $\Omega$

$$
\operatorname{Det}^{\prime}\left(\Omega^{-1} D \Omega\right)=\operatorname{Det}^{\prime}(D),
$$

since the eigenvalues $\lambda$ of $D$ and $\Omega^{-1} D \Omega$ coincide and $j_{D}(\lambda)=j_{\Omega^{-1} D \Omega}(\lambda)$.

We shall use in the next section the following result:

Lemma 4. For $\alpha$ small enough

$$
\left.\frac{d}{d s}\left[s K_{-s-1}(D+\alpha I ; x, x)\right]\right|_{s=0}=\left.\frac{d}{d s}\left[s K_{-s-1}(D+P+\alpha I ; x, x)\right]\right|_{s=0}+h_{\alpha}(x),
$$

where

$$
h_{\alpha}(x)=\sum_{n=1}^{N} \sum_{j=0}^{N}(-1)^{j}\left(\frac{1}{\alpha^{j+1}}-\frac{1}{(1+\alpha)^{j+1}}\right) D^{j} \phi_{n}(x) \otimes \phi_{n}^{\dagger}(x) .
$$

Proof. Note that for any invertible elliptic operator $L, K_{-s-1}(L ; x, x)$ has at the most a single pole at $s=0$ [2], then expression (2.4) makes sense.

For Res large enough, from Lemma 3, we can write

$$
\begin{aligned}
K_{-s-1}(D+\alpha I ; x, x)= & \sum_{n=1}^{\infty}(D+\alpha I)^{-s-1} \phi_{n}(x) \otimes \phi_{n}^{\dagger}(x) \\
= & K_{-s-1}(D+P+\alpha I ; x, x) \\
& +\sum_{n=1}^{N}\left[(D+\alpha I)^{-s-1}-(D+P+\alpha I)^{-s-1}\right] \phi_{n}(x) \otimes \phi_{n}^{\dagger}(x) .
\end{aligned}
$$

Then,

$$
\begin{aligned}
\left.\frac{d}{d s}\left[s K_{-s-1}(D+\alpha I ; x, x)\right]\right|_{s=0}= & \left.\frac{d}{d s}\left[s K_{-s-1}(D+P+\alpha I ; x, x)\right]\right|_{s=0} \\
& +\sum_{n=1}^{N}\left[(D+\alpha I)^{-1}-(D+P+\alpha I)^{-1}\right] \phi_{n}(x) \otimes \phi_{n}^{\dagger}(x),
\end{aligned}
$$

and this yields to (2.4). Q.E.D.

\section{An Application: Chiral Changes in Fermionic Variables}

Recently, Fujikawa [6] has shown that the Euclidean path-integral measure for gauge theories with fermions is not invariant under chiral transformations, and that it gives rise to a Jacobian related to the Adler-Bell-Jackiw anomaly [7]. In ref. [3], we studied this problem for invertible Dirac operators from the $\zeta$-function point of view. In this section we shall discuss how these results are modified when the Dirac operator is non-invertible. This occurs, for example, when fermions are coupled to a gauge field in a non-trivial topological sector. 
We start from the generating functional for a massless Dirac field coupled to arbitrary external fields

$$
Z(D)=\int \mathscr{D} \bar{\psi} \mathscr{D} \psi \exp \left\{-\int \bar{\psi} D \psi d x\right\},
$$

where the non-necessarily hermitian Euclidean Dirac operator is

$$
D=i \gamma_{\mu} \partial_{\mu}+g_{v} \gamma_{\mu} V_{\mu}+g_{A} \gamma_{5} \gamma_{\mu} A_{\mu}+g_{T}\left[\gamma_{\mu}, \gamma_{\nu}\right] T_{\mu \nu}+g_{S} S+g_{p} \gamma_{5} P,
$$

$V_{\mu}, A_{\mu}, T_{\mu \nu}, S$, and $D$ are vector, axial-vector, tensor, scalar, and pseudoscalar fields respectively, and can also belong to a representation of the algebra of a symmetry group $G$.

The Dirac matrices we are using satisfy $\left\{\gamma_{\mu}, \gamma_{v}\right\}=2 \delta_{\mu v}, \gamma_{\mu}^{+}=\gamma_{\mu}, \gamma_{5} \gamma_{\mu}+\gamma_{\mu} \gamma_{5}=0$, $\gamma_{5}^{2}=1$. Furthermore, we shall assume that the fields behave at infinity, so that it is possible to compactify $R^{n}$, for example, to $S^{n}$ by stereographic projection.

According to Berezin's integration rules [8], the path-integral in Eq. (3.1) is formally the determinant of the operator $D$. But, as it is well known, it either diverges or vanishes. It diverges for invertible $D$, and is zero if $D$ admits $\lambda=0$ as eigenvalue. However, it is necessary to obtain a regularized expression for $Z(D)$. This can be achieved by means of $\operatorname{Det}^{\prime}(D)$, i.e. projecting out the null space of $D$ and its powers when it has nilpotent elements, and regularizing the product of non-zero eigenvalues considered with their corresponding multiplicities. Note that according to (2.2), each eigenvalue $\lambda$ appears as many times as the dimension of the space of generalized eigenfunctions corresponding to $\lambda[4]$.

It is worth stressing that when $D$ has nilpotent elements, the path-integral in Eq. (3.1) does vanish if one does not project out the space $\mathcal{N}$ of nilpotent elements. Once this projection has been carried out the remaining path-integral precisely yields to the determinant of the restriction of $D$ to $\mathscr{N}^{\perp}$.

Therefore, we define the regularized generating functional $Z_{\text {reg }}(D)$ as

$$
Z_{\text {reg }}(D) \equiv \operatorname{Det}^{\prime}(D) \text {. }
$$

Note that there is no need for considering the hermitian semi-definite operator $D^{+} D$ in the definition of $Z_{\text {reg }}(D)$, as often appears in literature. Of course, this is necessary when the regularization is performed by means of the heat-kernel approach. However, it can be proved [9] that for hermitian operators both approaches yield to the same chiral Jacobian.

The $Z_{\mathrm{reg}}(D)$ defined in Eq. (3.3) is, of course, invariant under a unitary symmetry transformation of the theory

$$
D \rightarrow \Omega D \Omega^{-1},
$$

since according to Eq. (2.3), we have

$$
Z_{\text {reg }}\left(\Omega D \Omega^{-1}\right)=Z_{\text {reg }}(D) .
$$

If we perform, in the path-integral defining $Z(D)$, Eq. (3.1), the infinitesimal change of fermionic variables

where

$$
\psi=\Omega_{5} \chi, \quad \bar{\psi}=\bar{\chi} \Omega_{5},
$$

$$
\Omega_{5}=1+\varepsilon \gamma_{5} \phi(x)
$$


we shall obtain

$$
\operatorname{Det}^{\prime}(D)=J \operatorname{Det}^{\prime}\left(\Omega_{5} D \Omega_{5}\right),
$$

where $J$ is the "Jacobian" associated to the linear transformation (3.6).

We are going to denote $\tilde{L}$ as the transformed operator of an operator $L$ through the chiral infinitesimal transformation (3.6),

$$
L \rightarrow \tilde{L}=\Omega_{5} L \Omega_{5}
$$

We can write

$$
\tilde{D}+\alpha I=(D+\alpha I)+(\tilde{D}-D),
$$

where $\tilde{D}-D$ is a zero-order differential operator because the principal part of $D$ (i.e. $i \not$ ) anticommutes with $\gamma_{5}$. Then according to ref. [3].

$$
\operatorname{Det}(D+\alpha I)=J_{\alpha} \operatorname{Det}(\tilde{D}+\alpha I) \text {, }
$$

where $J_{\alpha}$ satisfies

$$
\ln J_{\alpha}=-\left.\varepsilon \frac{d}{d s}\left\{s \operatorname{Tr}\left[(D+\alpha I)^{-s-1}\left(\gamma_{5} \phi D+D \gamma_{5} \phi\right)\right]\right\}\right|_{s=0} .
$$

Since, for Res large enough, $(D+\alpha I)^{-s}$ is a trace-class operator, from the cyclicness of the trace,

$$
\begin{aligned}
\operatorname{Tr} & {\left[(D+\alpha I)^{-s-1}\left(\gamma_{5} \phi D+D \gamma_{5} \phi\right)\right] } \\
& =\operatorname{Tr}\left[(D+\alpha I)^{-s-1}\left(\gamma_{5} \phi(D+\alpha I)+(D+\alpha I) \gamma_{5} \phi-2 \alpha \gamma_{5} \phi\right)\right] \\
& =2 \operatorname{Tr}\left[(D+\alpha I)^{-s} \gamma_{5} \phi\right]-2 \alpha \operatorname{Tr}\left[(D+\alpha I)^{-s-1} \gamma_{5} \phi\right] .
\end{aligned}
$$

Now, using Lemma 4, we have

$$
\begin{aligned}
\left.\frac{d}{d s}\left\{s \operatorname{Tr}\left[(D+\alpha I)^{-s-1} \gamma_{5} \phi\right]\right\}\right|_{s=0}= & \left.\frac{d}{d s}\left\{s \operatorname{Tr}\left[(D+P+\alpha I)^{-s-1} \gamma_{5} \phi\right]\right\}\right|_{s=0} \\
& +\int \operatorname{tr}\left[h_{\alpha}(x) \gamma_{5} \phi\right] d x .
\end{aligned}
$$

So, replacing (3.13) and (3.14) in (3.12), we get

$$
\ln J_{\alpha}=-\left.\varepsilon\left\{\frac{d}{d s}\left[s\left(B_{\alpha}(s)-\alpha C_{\alpha}(s)\right)\right]\right\}\right|_{s=0}+2 \alpha \varepsilon F_{\alpha},
$$

with

Moreover

$$
\begin{aligned}
B_{\alpha}(s) & =2 \operatorname{Tr}\left[(D+\alpha I)^{-s} \gamma_{5} \phi\right], \\
C_{\alpha}(s) & =2 \int \operatorname{tr}\left[K_{-s-1}(D+P+\alpha I ; x, x) \gamma_{5}\right] \phi d x, \\
F_{\alpha} & =\int \operatorname{tr}\left[h_{\alpha}(x) \gamma_{5}\right] \phi d x .
\end{aligned}
$$

$$
\begin{aligned}
& -\left.\varepsilon \frac{d}{d s}\left[s B_{\alpha}(s)\right]\right|_{s=0}=\ln \widetilde{J}_{\alpha}, \\
& -\left.\varepsilon \frac{d}{d s}\left[s C_{\alpha}(s)\right]\right|_{s=0}=\ln \hat{J}_{\alpha},
\end{aligned}
$$


where $\tilde{J}_{\alpha}$ and $\hat{J}_{\alpha}$ are defined by

$$
\operatorname{Det}(D+\alpha I)=\widetilde{J}_{\alpha} \operatorname{Det} \widetilde{(D+\alpha I)}
$$

and

$$
\operatorname{Det}\left(D+P+\alpha I+\varepsilon \gamma_{5} \phi\right)=\hat{J}_{\alpha} \operatorname{Det}(D+P+\alpha I) .
$$

Thus, (3.12) can be written as

$$
\ln J_{\alpha}=\ln \tilde{J}_{\alpha}-\alpha \ln \hat{J}_{\alpha}+2 \alpha \varepsilon F_{\alpha} .
$$

From the continuity results of ref. [3], we see that

$$
\left.\lim _{\alpha \rightarrow 0} \varepsilon \frac{d}{d s}\left[s C_{\alpha}(s)\right]\right|_{s=0}=\ln \hat{J}\left(=\left.\ln \hat{J}_{\alpha}\right|_{\alpha=0}\right),
$$

and then the second term in the right-hand-side of (3.21) vanishes when $\alpha \rightarrow 0$.

We can compute $\ln \widetilde{J}_{\alpha}$ in terms of the kernel $K_{0}(D+\alpha I ; x, x)[2,3]$

$$
\ln \widetilde{J}_{\alpha}=-2 \varepsilon \int \operatorname{tr}\left[K_{0}(D+\alpha I ; x, x) \gamma_{5}\right] \phi d x,
$$

and use the fact that $K_{0}(D+\alpha I ; x, x)=K_{0}(D+P+\alpha I ; x, x)$, since $K_{0}$ depends only on the asymptotic expansion of the symbol of the operator, and this expansion does not change when the infinitely smoothing operator $P$ is added.

Then,

$$
\ln \widetilde{J}_{\alpha}=-2 \varepsilon \int \operatorname{tr}\left[K_{0}(D+P+\alpha I ; x, x) \gamma_{5}\right] \phi d x,
$$

so

$$
\lim _{\alpha \rightarrow 0} \ln \widetilde{J}_{\alpha}=-2 \varepsilon \int \operatorname{tr}\left[K_{0}(D+P ; x, x) \gamma_{5}\right] \phi d x .
$$

Note that for the computation of $K_{0}(D+P ; x, x)$, one can formally compute " $K_{0}(D ; x, x)$ " following Seeley's formulae disregarding the fact that $D$ is not invertible.

The last term in the right-hand-side of (3.21), $\alpha F_{\alpha}$, does not in general have a limit when $\alpha \rightarrow 0$, owing to the possible presence of negative powers of $\alpha$. The presence of such divergent terms indicates the fact that the number of nilpotent elements, $N$, changes through the transformation (3.9). This change leads to a discontinuity at $\varepsilon=0$ in $\operatorname{Det}^{\prime}(D)$.

If $N$ does not change through the transformation (3.9), continuity arguments imply that $\alpha F_{\alpha}$ has when $\alpha \rightarrow 0$ the limit

$$
\sum_{j=1}^{N} \int\left\langle\gamma_{5} \phi \phi_{j}, \phi_{j}\right\rangle d x
$$

Then,

$$
\ln J=\lim _{\alpha \rightarrow 0} \ln J_{\alpha}=-2 \varepsilon \int \operatorname{tr}\left[K_{0}(D+P ; x, x) \gamma_{5}\right] \phi d x+\sum_{j=1}^{N} \int\left\langle\gamma_{5} \phi \phi_{j}, \phi_{j}\right\rangle d x .
$$

Of course, $N$ does not change if $\operatorname{Ker} D=\operatorname{Ker} D^{2}$. This is the case, for instance, for any normal operator. 
Another interesting application of (3.27) arises when $D$ is such that $D \gamma_{5}=-\gamma_{5} D$, which implies $\tilde{D}=D$ for a global chiral transformation, i.e. $\phi=$ const, and then $\ln J=0$. In this case, from (3.27) we get

$$
\sum_{j=1}^{N} \int\left\langle\gamma_{5} \phi_{j}, \phi_{j}\right\rangle d x=\int \operatorname{tr}\left[K_{0}(D+P ; x, x) \gamma_{5}\right] d x .
$$

It is easy to see that the basis of $\mathcal{N},\left\{\phi_{j}\right\}_{j=1, \ldots, N}$, can be chosen in such a way that $\gamma_{5} \phi_{j}= \pm \phi_{j}$. Then, we find

$$
N_{+}-N_{-}=\int \operatorname{tr}\left[K_{0}(D+P ; x, x) \gamma_{5}\right] d x,
$$

where $N_{ \pm}$is the number of eigenfunctions $\phi_{j}$ of $\gamma_{5}$ with eigenvalues \pm 1 . Therefore, the integral in the right-hand side (3.29) is an integer number.

When $D$ is also hermitian, taking into account that $K_{0}(D ; x, x)=K_{0}\left(D^{2} ; x, x\right)$ [9], expression (3.29) reduces to the usual index formula.

Acknowledgements. We are very grateful to R. Rodriguez for helpful discussion and to Prof. R. T. Seeley for valuable correspondence.

\section{References}

1. Hawking, S.W.: Zeta function regularization of path integrals in curved spacetime. Commun. Math. Phys. 55, 133-148 (1977)

2. Seeley, R.T.: Complex powers of an elliptic operator. Am. Math. Soc. Proc. Sym. Pure Math. 10, 288-307 (1967)

3. Gamboa-Saraví, R.E., Muschietti, M.A., Solomin, J.E.: On perturbation theory for regularized determinants of differential operators. Commun. Math. Phys. 89, 363-373 (1983)

4. Agmon, S.: Lectures on elliptic boundary value problems. New York: Van Nostrand Company, Inc. 1965

5. Calderón, A.P.: Lecture Notes on pseudo-differential operators and elliptic boundary value problems, I. Instituto Argentino de Matemática, (1976)

6. Fujikawa, K.: Path integral for gauge theories with fermions. Phys. Rev. D21, 2848 (1980)

7. Adler, S.: Axial-vector vertex in spinor electrodynamics, Phys. Rev. 177, 2426 (1969); Bell, J., Jackiw, R.: A PCAC Puzzle: $\pi^{0} \rightarrow \gamma \gamma$ in the $\sigma$-Model. Nuovo Cimento 60 A, 47 (1969)

8. Berezin, F.: The method of second quantization. New York: Academic Press 1969

9. Gamboa-Saraví, R.E., Muschietti, M.A., Schaposnik, F.A., Solomin, J.E.: Chiral symmetry and functional integral. La Plata University preprint (1983). Ann. Phys. (in press)

Communicated by H. Araki

Received November 7, 1983 
